\title{
Factors associated with catastrophic total costs due to tuberculosis under a designated hospital service model: a cross- sectional study in China
}

\author{
Tianchi Yang ${ }^{1 *}$ (D), Tong Chen ${ }^{1}$, Yang Che ${ }^{1}$, Qin Chen ${ }^{2}$ and Dingyi Bo ${ }^{3}$
}

\begin{abstract}
Background: Certain districts and counties in China designated local general hospital as the designated hospital for tuberculosis (TB) management after the promulgation of the Law of Practicing Physicians in 2009. To our knowledge, there is limited research on catastrophic payments of TB patients under this service model, often with inconsistent conclusions. In addition, there has been no published studies from China using the updated 2018 World Health Organization (WHO) definition of catastrophic total costs due to TB. This study used the latest criterion recommended by the WHO to analyze the incidence of catastrophic total costs for households affected by TB under the designated hospital model and explore its influencing factors.

Methods: A cross-sectional analysis was carried out in all ten designated hospitals in Ningbo, China. Eligible pulmonary TB cases confirmed by sputum culture of Mycobacterium tuberculosis were recruited and surveyed from September 2018 to October 2018. We evaluated catastrophic total costs using total costs for TB treatment exceeding $20 \%$ of the household's annual pre-TB income. A sensitivity analysis was performed while varying the thresholds. The least absolute shrinkage and selection operator (LASSO) regression were applied to select variables, and multiple logistic regression analysis were used to identify the determinants of catastrophic total costs.

Results: A total of 672 patients were included, with a median age of 41 years old. The rate of catastrophic total costs of surveyed households was 37.1\%, and that of households affected by MDR was 69.6\%. Medical cost accounted for more than $60 \%$ of the total cost. $57.7 \%$ cases were hospitalized. The hospitalization rates of patients with no comorbidities, no severe adverse drug reactions, and rifampin-sensitive TB were 53.9, 54.9, and 55.3\%, respectively. Patients in the poorest households had the highest hospitalization rates (Q1:54.8\%, Q2:61.4\%, Q3:52.2\%, Q4:49.5\%, Q5:69.7\%, $P=0.011)$ and the highest incidence of severe adverse drug reactions (Q1:29.6\%, Q2:19.6\%, Q3: 28.0\%, Q4:33.7\%, Q5:35.3\%, $P=0.034$ ). Factors such as elderly, minimum living security, unemployed before or after illness, poor economic status, seeking medical care outside the city, hospitalization, absence of local basic medical insurance coverage and MDR were positively associated with catastrophic costs.

(Continued on next page)
\end{abstract}

\footnotetext{
* Correspondence: cn-yangtc@outlook.com

'Institute of Tuberculosis Prevention and Control, Ningbo Municipal Center

for Disease Control and Prevention, Ningbo, Zhejiang, China

Full list of author information is available at the end of the article
}

C C The Author(s). 2020 Open Access This article is licensed under a Creative Commons Attribution 4.0 International License, which permits use, sharing, adaptation, distribution and reproduction in any medium or format, as long as you give appropriate credit to the original author(s) and the source, provide a link to the Creative Commons licence, and indicate if changes were made. The images or other third party material in this article are included in the article's Creative Commons licence, unless indicated otherwise in a credit line to the material. If material is not included in the article's Creative Commons licence and your intended use is not permitted by statutory regulation or exceeds the permitted use, you will need to obtain permission directly from the copyright holder. To view a copy of this licence, visit http://creativecommons.org/licenses/by/4.0/ The Creative Commons Public Domain Dedication waiver (http://creativecommons.org/publicdomain/zero/1.0/) applies to the data made available in this article, unless otherwise stated in a credit line to the data. 


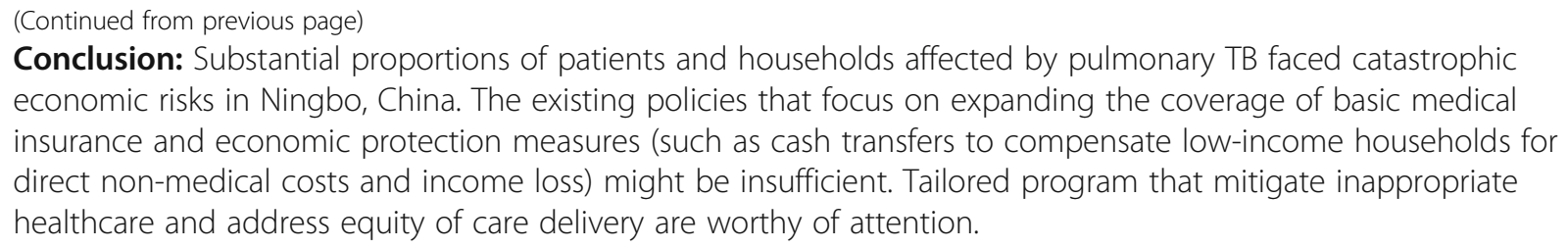

Keywords: Tuberculosis, Catastrophic total costs, Designated hospital, Factors

\section{Background}

Tuberculosis (TB) is one of the major worldwide infectious diseases, and it remains an urgent public health issue in developing countries [1]. Poverty increases the risk of $\mathrm{TB}$, impacts of $\mathrm{TB}$ on wage and earning power further exacerbates impoverishment [2, 3]. Since 2005, the Chinese government has implemented a free TBcare policy that all pulmonary TB patients are provided with free sputum smear tests, chest X-ray examinations, and anti-TB drugs $[4,5]$. Theoretically, patients do not need to pay out-of-pocket expenses for the diagnosis and treatment of TB.

The "Law of Practicing Physicians" promulgated by Chinese government in 2009 stipulates that public health doctors are not qualified to provide clinical care. In response to the regulations, each administrative district or county has designated a general hospital to set up a TB clinic to diagnose and treat TB within its jurisdiction. Other hospitals transfer suspected TB cases to designated hospitals, and the institutions for disease control and prevention no longer provide $\mathrm{TB}$ clinical services. TB patients treated at designated hospitals expressed concerns on the high level of out-of-pocket expenses after the service model changed [5-7]. Some patients interrupted or discontinued standardized treatment due to cost concerns [6].

To date, there have been few studies on catastrophic health expenditures (CHE) of TB patients under the designated hospital model in China, and the conclusions are not consistent $[8,9]$. For example, Zhou C, etc. found that hospitalization is an important determinant of the occurrence of CHE [9], while in a study by Duan et al., despite observed strong association between hospitalization and CHE, the association was not statistically significant after adjusting for potential confounding factors (aOR:1.342, 95\%CI: 0.950-1.897) [8]. The conflicting findings from two studies could probably resulted from the rates of patients participating in the New Cooperative Medical Scheme, an insurance specifically designed to cover more disadvantaged rural population. Because place of residence and type of health insurance scheme are highly confounded with inpatient service utilization patterns, the difference in insurance status distributions likely caused conflicting results $[8$, 9]. However, Li X, etc. pointed out that despite the limited benefit, patients participating in this scheme had a lower incidence of CHE [10]. In addition, since the World Health Organization (WHO) proposed catastrophic total costs, that is, total costs exceeding $20 \%$ of a household's pre-TB annual income as the criteria for catastrophic payments in 2018, no relevant research reports from China have been seen.

Here, this study used the latest criterion recommended by the WHO to analyze the incidence of catastrophic total costs for households affected by TB under the designated hospital model, and explore its influencing factors.

\section{Methods}

\section{Study sites}

This study was conducted in ten counties of Ningbo Municipality, China. Ningbo is one of the most economically developed cities on the east coast of China, near Shanghai, with a population of 5.7 million permanent residents and 4.3 million floating population. There are ten designated hospitals for $\mathrm{TB}$ in the city, covering every district and county. The policy stipulated that from January 2016, all active TB patients and suspected cases were required to be referred to TB-designated hospitals for treatment. In 2017, the annual TB notification rate in Ningbo was 48.9 per 100,000, at a lower-middle level in the country.

According to the requirements of the National Tuberculosis Control Program (NTCP), pulmonary tuberculosis cases and suspected cases are diagnosed and treated in designated hospitals. Pulmonary tuberculosis treatment is carried out in accordance with the chemotherapy regimen recommended by the WHO or the personalized regimen made by clinicians. Generally, outpatient treatment is adopted, but complicated $\mathrm{TB}$ cases, patients with severe comorbidities or serious adverse drug reactions, addition to MDR cases required TB care under in-patient settings. MDR patients are referred to the municipal designated hospital for treatment, and are generally required to be hospitalized during the intensive period until the sputum culture 
turns negative. All patients are followed up regularly by community doctors throughout the treatment, including the intensive period once every 10 days and the continuation period once a month. During each visit, the community doctor will collect the patient's medical expenses receipts, which are used to determine the amount of subsidies to the patients after treatment.

\section{Study design}

A cross-sectional survey was carried out in all ten counties of Ningbo from September 1 to October 31, 2018. Due to the fact that extrapulmonary TB and tuberculous pleurisy were not covered by the NTCP during the study period, and there were overdiagnosis and misdiagnosis of clinically diagnosed cases, the study included only pulmonary TB cases confirmed by sputum culture. The pilot survey showed that the prevalence of catastrophic costs due to TB was about $40 \%$. The study set a relative precision of $0.1, \alpha$ error of 0.05 , and an expected nonresponse rate of $10 \%$. Therefore, the minimum sample size was 550 . Until reaching our predetermined sample size, we consecutively recruited all sputum culturepositive pulmonary $\mathrm{TB}$ cases that met the following inclusion criteria from ten designated hospitals: (1) registered in designated hospitals in Ningbo between January 1, 2017, and December 31, 2017; (2) patients with rifampicin-susceptible TB who had finished treatment or patients with rifampicin-resistant TB who had been treated for at least 12 months.

\section{Data collection}

Trained investigators interviewed each participant face-toface using a modified version of the WHO patient cost tool [11]. The questionnaire included the demographic and socioeconomic status (age, gender, education, health insurance, household income and expenditure, etc.), health care seeking behavior (hospitalization, medical care outside the city, patient/diagnosis delay, etc.), clinical features (type of disease, adverse drug reactions and comorbidities, etc.), costs of TB diagnosis and treatment (medical cost, direct non-medical cost and income loss).

Direct medical costs refer to out-of-pocket (OOP) payments for medical services (consultation, medicine, and other medical procedures), net of any reimbursements. Direct non-medical costs refer to OOP payments related to accommodation, transportation, and food etc., net of any reimbursements. Indirect costs refer to income loss of a household caused by TB during the TB episode, estimated by self-reported household annual pre-TB income minus self-reported household annual income during TB treatment. The costs were ascertained from the onset of TB symptoms (the occurrence of cough, sputum production more than 2 weeks, or hemoptysis, blood-tinged sputum, fever or chest pain, etc.) to the end of treatment or investigation. Medical costs came from medical receipts collected by community doctors. The self-report was used to collect direct non-medical expenses and indirect costs, as well as a household income and living expenses. Costs and incomes were converted to United States Dollars (US\$) using the average currency exchange rate during the study period (US\$1 = 6.75 Chinese Yuan).

\section{Definitions \\ Household's annual income}

A household's annual income refers to the total income of all members of a household for 1 year. The household's annual pre-TB income means the household annual income in the year before diagnosis with TB.

\section{Household's annual capacity to pay}

A household's annual capacity to pay is the total annual income of a household minus the cost of a household's annual food consumption.

\section{Catastrophic total costs due to TB}

Catastrophic total costs due to TB is defined as total costs for TB treatment (the sum of direct and indirect costs for the entire TB episode) exceeding $20 \%$ of the household's annual pre-TB income.

\section{Catastrophic health expenditure}

CHE is defined OOP medical expenses for TB care exceeding a specified proportion of the household income or capacity to pay. There is no consensus on the threshold $[9,12]$. In this study, we estimated CHE using two most commonly definitions: (a) annual OOP medical expenses over $40 \%$ of the household's annual capacity to pay during TB treatment, and (b) annual OOP medical expenses over $10 \%$ of the household's annual income during TB treatment $[9,12,13]$.

\section{Basic medical insurance}

Basic medical insurance is a social insurance system implemented by the government to compensate the economic losses caused by the disease risk of the insured. There are two types in Ningbo, one is the basic medical insurance for urban employees, which is mandatory and provided to people with a legitimate occupation. The other is the basic medical insurance for urban and rural residents, which is voluntary and covers mainly those under 18 years old, unemployed, and rural residents. Both are only valid at the place of enrollment.

\section{Rifampicin-susceptible (RS) pulmonary TB}

RS pulmonary TB is confirmed by sputum culture of Mycobacterium tuberculosis, and clinical isolates are 
susceptible to rifampicin in an in vitro anti-TB drug sensitivity test.

\section{Rifampicin-monoresistant (RMR) pulmonary TB}

RMR pulmonary TB is confirmed by sputum culture of Mycobacterium tuberculosis, and clinical isolates are resistant to rifampicin and susceptible to isoniazid in an in vitro anti-TB drug sensitivity test.

\section{Multidrug-resistant (MDR) pulmonary TB}

MDR pulmonary TB is confirmed by sputum culture of Mycobacterium tuberculosis, and clinical isolates are resistant to both rifampicin and isoniazid in an in vitro anti-TB drug sensitivity test.

\section{Statistical analysis}

Data were double entered and checked using EPI Data V3.1. Continuous variables were presented as means (standard deviation, SD) and medians (interquartile range, IQR), while categorical variables were described as absolute numbers and frequencies. Using univariate logistic regression analysis, the association between different factors and catastrophic total costs was examined by estimating odd ratios (ORs) with $95 \%$ confidence intervals (CIs). We used the least absolute shrinkage and selection operator (LASSO) regression for variable selection, and ten-fold cross-validation together with the 1SE rule were used to select the predictive variables from the primary data set. Multivariable analyses of selected variables related to catastrophic total costs were conducted using the logistic regression analysis. We addressed the interaction between hospitalization and severe adverse drug reaction by including the interaction term in a supplemental multivariable adjusted model. Adjusted odds ratios (aORs) and 95\% confidence intervals were derived from the final model. The level of statistical significance was set at 5\%. All analyses were performed using the statistical software R (http://www.R-project.org) and Empower (R) (www.empowerstats.com, X\&Y Solutions, Inc., Boston, MA).

\section{Sensitivity analyses}

We varied the threshold $(20 \% \pm 5 \%)$ to observe how this would affect the percentage of households incurring catastrophic total costs. We also compared the incidence of catastrophic total costs with different thresholds to the incidence of catastrophic health expenditures with two common definitions. Finally, a supplemental model was conducted including an interaction term between hospitalization and severe adverse drug reactions.

\section{Results}

Characteristics of participants and their households

A total of 683 patients were qualified, of whom 11 refused to participate. Thus, the analysis finally included 672 patients, of which $586(87.2 \%)$ cases were RS, 30 (4.5\%) were RMR, and 56 (8.3\%) were MDR. Table 1 shows the basic characteristics and hospitalization of the participants. The average age of participants was 43 years, with a median age of 41 years. Approximately $90 \%$ of patients received at least primary school education. About one-third of patients were floating population and did not have health insurance where they lived or worked. Less than $5 \%$ of households lived below the poverty line in Ningbo. According to the World Bank's poverty line standard of US \$ 1.90 per day, $4.2 \%$ (28/ 672) of the households surveyed before suffering from TB were in poverty, and this proportion rose to $15.5 \%$ $(104 / 672)$ after TB. The proportion of patients who were employed before illness was $62.8 \%$, and after illness was $57.4 \%$. Patients in the poorest households had the highest the hospitalization rates (Q1:54.8\%, Q2:61.3\%, Q3: 52.2\%, Q4:49.5\%, Q5:69.7\%, $P=0.011$ ) and the highest incidence of severe adverse drug reactions (Q1:29.6\%, $\mathrm{Q} 2: 19.6 \%, \mathrm{Q}: 28.0 \%, \mathrm{Q} 4: 33.7 \%, \mathrm{Q}: 35.3 \%, P=0.034)$.

Total costs due to TB and its share of household's pre$\mathrm{TB}$ annual income among patients with RS, RMR and MDR were significantly different, but there was no statistical difference in the household's pre-TB annual income. Among RS, RMR and MDR patients, the medical cost accounted for more than $60 \%$ of the total cost (Table 2).

Whether it was direct medical expenses or out-ofpocket medical expenses, inpatients were significantly higher than non-inpatients (Table 3).

\section{Catastrophic total costs due to TB}

Among the surveyed households, over 37\% of households spent at least 20 of their household's pre-TB annual income on pulmonary TB diagnosis and treatment. Table 4 presents how the incidence of catastrophic total costs changes across different thresholds. When the threshold varied from $25 \%$ of a household's pre-TB annual income to $15 \%$, the rate of catastrophic total costs increased rapidly from 30.2 to $46.0 \%$. Regardless of the threshold, households affected by MDR always had the highest catastrophic cost rate, followed by RR and RS. The catastrophic cost rate calculated by using total costs over $15 \%$ or $20 \%$ of a household's pre-TB annual income was close to the CHE rate measured by using OOP medical expenses over $40 \%$ of a household's annual ability to pay during TB treatment.

\section{The distributions of catastrophic total costs}

In univariate logistic regression, many variables were statistically related to catastrophic total costs: age, 
Table 1 Sociodemographic and clinical characteristics of the study population, by hospitalization status and overall

\begin{tabular}{|c|c|c|c|c|}
\hline Variables & Number of participants (\%) & Hospitalisation (\%) & Non-hospitalisation (\%) & $P$-value* \\
\hline Total participants & $672(100)$ & $388(57.7)$ & $284(42.3)$ & \\
\hline Age (years) & & & & 0.030 \\
\hline$<60$ & $514(76.5)$ & $285(55.4)$ & $229(44.6)$ & \\
\hline$\geq 60$ & $158(23.5)$ & $103(65.2)$ & $55(34.8)$ & \\
\hline Gender & & & & 0.376 \\
\hline Male & $432(64.3)$ & $244(56.5)$ & $188(43.5)$ & \\
\hline Female & $240(35.7)$ & $144(60.0)$ & $96(40.0)$ & \\
\hline Education & & & & 0.927 \\
\hline None & $70(10.4)$ & $41(58.6)$ & $29(41.4)$ & \\
\hline Primary or Junior school & $368(54.8)$ & $210(57.1)$ & $158(42.9)$ & \\
\hline Senior school or above & $234(34.8)$ & $137(58.5)$ & $97(41.5)$ & \\
\hline Floating population & & & & 0.004 \\
\hline No & $437(65.0)$ & $270(61.8)$ & $167(38.2)$ & \\
\hline Yes & $235(35.0)$ & $118(50.2)$ & $117(49.8)$ & \\
\hline Minimum living security household & & & & 0.355 \\
\hline No & $640(95.2)$ & $367(57.3)$ & $273(42.7)$ & \\
\hline Yes & $32(4.8)$ & $21(65.6)$ & $11(34.4)$ & \\
\hline Residence & & & & 0.486 \\
\hline Rural & $316(47.0)$ & $178(56.3)$ & $138(43.7)$ & \\
\hline Urban & $356(53.0)$ & $210(59.0)$ & $146(41.0)$ & \\
\hline Employment status before illness & & & & 0.002 \\
\hline Employed & $422(62.8)$ & $224(53.1)$ & $198(46.9)$ & \\
\hline Unemployed & $250(37.2)$ & $164(65.6)$ & $86(34.4)$ & \\
\hline Employment status after illness & & & & $<0.001$ \\
\hline Employed & $386(57.4)$ & $250(64.8)$ & $136(35.2)$ & \\
\hline Unemployed & $286(42.6)$ & $138(48.3)$ & $148(51.7)$ & \\
\hline Local basic medical insurance & & & & 0.024 \\
\hline No & $212(31.5)$ & $109(51.4)$ & $103(48.6)$ & \\
\hline Yes & $460(68.5)$ & $279(60.7)$ & $181(39.3)$ & \\
\hline Household economic status & & & & 0.011 \\
\hline Q1 & $135(20.1)$ & $74(54.8)$ & $61(45.2)$ & \\
\hline Q2 & $163(24.3)$ & $100(61.3)$ & $63(38.7)$ & \\
\hline Q3 & $157(23.4)$ & $82(52.2)$ & $75(47.8)$ & \\
\hline Q4 & $95(14.1)$ & $47(49.5)$ & $48(50.5)$ & \\
\hline Q5 & $122(18.2)$ & $85(69.7)$ & $37(30.3)$ & \\
\hline Patient delay & & & & 0.263 \\
\hline No & $312(46.4)$ & $173(55.4)$ & $139(44.6)$ & \\
\hline Yes & $360(53.6)$ & $215(59.7)$ & $145(40.3)$ & \\
\hline Diagnosis delay & & & & 0.196 \\
\hline No & $583(86.8)$ & $331(56.8)$ & $252(43.2)$ & \\
\hline Yes & $89(13.2)$ & $57(64.0)$ & $32(36.0)$ & \\
\hline Type of case & & & & 0.002 \\
\hline New or never treated & $601(89.4)$ & $335(55.7)$ & $266(44.3)$ & \\
\hline Relapse or previously treated & $71(10.6)$ & $53(74.6)$ & $18(25.4)$ & \\
\hline
\end{tabular}


Table 1 Sociodemographic and clinical characteristics of the study population, by hospitalization status and overall (Continued)

\begin{tabular}{llll}
\hline Variables & Number of participants (\%) & Hospitalisation (\%) & Non-hospitalisation (\%) \\
\hline Comorbidities & & & $P$-value* \\
No & $584(86.9)$ & $315(53.9)$ & $269(46.1)$ \\
Yes & $88(13.1)$ & $73(83.0)$ & $15(17.0)$ \\
Seeking medical care outside the city & & & $262(44.6)$ \\
No & $587(87.4)$ & $325(55.4)$ & $22(25.9)$ \\
Yes & $85(12.6)$ & $63(74.1)$ & \\
Severe adverse drug reactions & & & $217(45.1)$ \\
No & $481(71.6)$ & $264(54.9)$ & $67(35.1)$ \\
Yes & $191(28.4)$ & $124(64.9)$ & \\
Drug sensitivity test & & $324(55.3)$ & $262(44.7)$ \\
RS & $586(87.2)$ & $20(66.7)$ & $10(33.3)$ \\
RMR & $30(4.5)$ & $44(78.6)$ & $12(21.4)$ \\
MDR & $56(8.3)$ & 0.018
\end{tabular}

Note: Floating population refers to a group of migrants without Hukou registered in the place where they live or work

Minimum living security household is defined as a household's anuual income per capita lower than US\$ 1429 . The designation of minimum living security household status needs to be certified by the local civil affairs bureau

Local basic medical insurance refers to the basic medical insurance registered in Ningbo city

Household economic status is ranked based on a household's annual income per capita, with quartile 1 (Q1) being the richest and quartile 5 (Q5) being

the poorest

Patient delay is determined as a time interval from the first onset of tuberculosis symptoms to the first visit to any health provider lasts for $>14$ days

Diagnostic delay is defined as a time interval between a patient's first visit to any health provider and the final diagnosis as TB lasts for $>14$ days

Comorbidities refers to the simultaneous occurrence of malignancy, diabetes mellitus, renal disease, etc.

$R S$ rifampicin-susceptible; $R M R$ rifampicin-monoresistant; $M D R$ multidrug-resistant

*the Chi-square tests were applied to assess differences between categories of variables

education, minimum living security household, employment status before illness, employment status after illness, household economic status, patient delay, type of case, comorbidities, seeking medical care outside the city, hospitalization, severe adverse drug reactions, drug sensitivity test result (Table 5).

\section{Factors associated with catastrophic total costs}

Based on nonzero coefficients in the LASSO regression model, thirteen possible variables affecting catastrophic total costs were included into the multivariable logistic regression model. In the adjusted model that controlled for confounding variables, the factors such as over 60 years old, minimum living security, unemployed before or after illness, poor economic status, seeking medical care outside the city, hospitalization, severe adverse drug reactions and MDR were positively associated with catastrophic costs. Local basic medical insurance was negatively related to catastrophic costs. Although preselected by LASSO to be related to catastrophic total costs, the factors "education", "relapse or previously treated" and "comorbidities" were not found to be significant at the $5 \%$ level in the logistic regression model. We acknowledge the potential interaction between hospitalization and adverse drug reactions based on the theoretical framework, therefore we included the term into our final model to improve the model fit. The results indicated that, except for adverse drug reactions, the others were similar to the original findings (Table 6).

\section{Discussion}

Putting an end to the catastrophic total costs due to TB is one of the three goals of the End TB strategy by WHO $[2,3,14]$. In this study examining the catastrophic total costs due to $\mathrm{TB}$ among patients treated in the NTCP network under a designated hospital service model, we found that more than one-third of patients and their households who were diagnosed and treated in designated hospitals face financial disasters due to TB, especially those with MDR. The incidence of catastrophic costs in this survey is lower than those of Ghana [15] and Vietnam [16], and is similar to the results of studies in Indonesia [17], Peru [18], and southern India [19].

We identified several independent factors associated with catastrophic total costs in this study, these factors could have influenced healthcare costs through different mechanisms. For example, patients over 60 years old, unemployment before illness, minimum living security status and lower income households may correlate with household's annual pre-TB income. On the other hand, lack of basic medical insurance coverage, seeking medical treatment outside the city, hospitalization and MDR affect direct costs, and unemployment after illness are associated with income losses. These findings 
Table 2 Total costs due to TB and household economic status, by drug susceptibility test

\begin{tabular}{|c|c|c|c|c|}
\hline Variables & RS & RMR & MDR & $P$-value* \\
\hline \multicolumn{5}{|c|}{ Total costs due to TB (US\$) } \\
\hline Mean (SD.) & $2094.5(2676.7)$ & $7669.1(9649.4)$ & $10,802.1(10,219.4)$ & \\
\hline Median(p25,p75) & $1377.8(740.72503 .7)$ & $3622.2(1159.312029 .6)^{a}$ & $7355.6(3129.614255 .6)^{a}$ & $<0.001$ \\
\hline \multicolumn{5}{|c|}{ Medical costs share of total costs (\%) } \\
\hline Mean (SD.) & $67.7(24.4)$ & $75.6(24.1)$ & $64.7(31.1)$ & \\
\hline Median(p25,p75) & $70.8(53.8,87.2)$ & $79.9(63.3,94.5)$ & $75.7(52.9,87.5)$ & 0.155 \\
\hline \multicolumn{5}{|c|}{ Direct non-medical costs share of total costs (\%) } \\
\hline Mean (SD.) & $25.4(22.7)$ & $20.4(27.6)$ & $27.9(30.7)$ & \\
\hline Median(p25,p75) & $19.9(8.5,34.6)$ & $8.2(3.1,24.4)$ & $15.6(8.6,35.1)$ & 0.059 \\
\hline \multicolumn{5}{|c|}{ Indirect costs share of total costs (\%) } \\
\hline Mean (SD.) & $7.7(13.7)$ & $6.9(8.7)$ & $11.0(18.5)$ & \\
\hline Median(p25,p75) & $0.0(0.0,11.4)$ & $2.9(0.0,13.9)$ & $2.9(0.0,14.7)$ & 0.118 \\
\hline \multicolumn{5}{|c|}{ household's pre-TB annual income (US\$) } \\
\hline Mean (SD.) & $14,375.6(10,762.1)$ & $14,732.3(6863.8)$ & $14,510.6(14,399.1)$ & \\
\hline Median(p25,p75) & $11,851.9(7407.417777 .8)$ & $14,814.8(8692.620729 .6)$ & $11,918.5(8114.815633 .3)$ & 0.446 \\
\hline \multicolumn{5}{|c|}{ Total costs share of household's pre-TB annual income (\%) } \\
\hline Mean (SD.) & $21.6(24.6)$ & $29.5(32.9)$ & $46.3(34.1)$ & \\
\hline Median(p25,p75) & $12.8(5.7,26.8)$ & $12.3(5.2,50.1)$ & $42.3(14.0,74.4)^{a, b}$ & $<0.001$ \\
\hline
\end{tabular}

Note: US\$: United States dollar

A currency exchange rate of Chinese RMB 675 Yuan to US\$ 100 Yuan (at the end of 2017)

$R S$ rifampicin-susceptible

$R M R$ rifampicin-monoresistant

$M D R$ multidrug-resistant

*Kruskal Wallis test

Pairwise comparisons were performed by post-hoc analyses with Bonferroni adjustment ( 3 comparisons: $P$-value< 0.017$)$

a significant compared to RS

bignificant compared to RMR

highlighted that in addition to reducing the direct costs of TB care for patients, especially those with MDR and severe adverse drug reactions [15], other financial protection measures such as compensation for income losses were needed to prevent low-income households from incurring catastrophic costs following TB diagnosis $[2,14,16]$.

Consistent with studies in other countries [17-19], there was an obviously negative correlation between the household's economic status and the occurrence of catastrophic total costs, that is, financially vulnerable households were more likely to suffer catastrophic costs. This prompted us to think about the drawbacks of the balanced subsidy policy. While the governments distribute TB subsidies the restricted budget every year, "one-sizefit-all" subsidies ensure the fairness of the program, but it ignored the disparate financial vulnerability among patients and households with TB $[9,14,20,21]$. Meanwhile, a more precise, tailored financial support might play a better role in poverty alleviation by minimizing the rate of catastrophic costs. Public subsidies programs often face budgetary constraints, a differentiated subsidy policy based on the household's economic status has more potential prioritizing protection to the poor against catastrophic costs.

According to the recommendations of the WHO and China's National TB Control Program, outpatient treatment is usually advocated for non-severe and/or nonMDR cases [22]. Nevertheless, the survey found that more than half of the patients were hospitalized during diagnosis and treatment, which is in line with other studies conducted in China [9, 23]. Previous studies have shown that hospitalization will lead to a substantial increase in the cost of treatment, and hospitalization cases face higher economic risks than outpatient cases $[6,22]$. In this study, hospitalization was a key factor influencing catastrophic costs, and its impact was second only to the household economic status. In recent years, as the responsibility for TB care has shifted from the Center for Disease Control and Prevention (CDC) to general hospitals, the proportion of patients hospitalized has increased significantly $[9,24,25]$. A literature search indicated that unnecessary hospitalization for TB is common in China, which is considered to be closely related to the profitseeking behavior of the hospital $[5,6,26-28]$. This behavior also reasonably explains that the direct medical 
Table 3 Direct medical expenses and medical costs, by hospitalization

\begin{tabular}{|c|c|c|c|}
\hline Variables & Hospitalisation & Non-hospitalisation & $P$-value* \\
\hline \multicolumn{4}{|l|}{$\mathrm{RS}$} \\
\hline \multicolumn{4}{|c|}{ Direct medical expenses (US\$) } \\
\hline Mean (SD.) & $3241.7(3240.4)$ & $1065.0(1125.8)$ & \\
\hline Median(p25,p75) & $2437.0(1670.43407 .4)$ & $859.3(563.0,1255.6)$ & $<0.001$ \\
\hline \multicolumn{4}{|l|}{ Medical costs (US\$) } \\
\hline Mean (SD.) & $2094.7(2623.8)$ & $703.4(994.9)$ & \\
\hline Median(p25,p75) & $1422.2(825.92303 .7)$ & $518.5(296.3959 .3)$ & $<0.001$ \\
\hline \multicolumn{4}{|l|}{ RMR } \\
\hline \multicolumn{4}{|c|}{ Direct medical expenses (US\$) } \\
\hline Mean (SD.) & 11,739.3 (8984.6) & $2268.1(2949.9)$ & \\
\hline Median(p25,p75) & 11,133.3 (4996.316174.1) & $1540.7(488.92440 .7)$ & $<0.001$ \\
\hline \multicolumn{4}{|l|}{ Medical costs (US\$) } \\
\hline Mean (SD.) & $8460.0(9204.2)$ & $983.7(971.3)$ & \\
\hline Median(p25,p75) & $4311.1(2525.912111 .1)$ & $763.0(92.61825 .9)$ & $<0.001$ \\
\hline \multicolumn{4}{|l|}{ MDR } \\
\hline \multicolumn{4}{|c|}{ Direct medical expenses (US\$) } \\
\hline Mean (SD.) & 12,559.6 (8252.9) & $1363.0(1033.6)$ & \\
\hline Median(p25,p75) & 11,348.1 (7103.716874.1) & $1688.9(511.11888 .9)$ & $<0.001$ \\
\hline \multicolumn{4}{|l|}{ Medical costs (US\$) } \\
\hline Mean (SD.) & $9666.3(8691.5)$ & $855.6(1033.3)$ & \\
\hline Median(p25,p75) & 7948.1 (3455.613151.9) & $348.1(0.0,1533.3)$ & $<0.001$ \\
\hline
\end{tabular}

Note: US\$: United States dollar

A currency exchange rate of Chinese RMB 675 Yuan to US\$ 100 Yuan (at the end of 2017)

$R S$ rifampicin-susceptible

$R M R$ rifampicin-monoresistant

MDR multidrug-resistant

*Mann-Whitney test

costs borne by patients receiving treatment in designated hospitals in Ningbo were much higher than those reported in other countries $[16,17,19]$, whether they were absolute values or their share of total costs.

Our findings have at least three policy implications. First, the existing "zero OOP" free TB care policy developed under the CDC service model implemented since the 1990s was insufficient in addressing the problem of catastrophic costs faced by patients. As our findings indicated, approximately $40 \%$ of individuals and their households incurred catastrophic spending due to TB. Secondly, we documented inefficiency in TB care delivery system. Despite adoption of economic protection interventions such as universal health coverage and cash

Table 4 Comparison of the incidence of catastrophic total costs and CHE in PTB-affected households at different thresholds

\begin{tabular}{|c|c|c|c|c|c|}
\hline \multirow[t]{2}{*}{ Groups } & \multicolumn{3}{|c|}{ the incidence of catastrophic total costs at the threshold } & \multirow[t]{2}{*}{ CHE1 } & \multirow[t]{2}{*}{ CHE2 } \\
\hline & $15 \%$ & $20 \%$ & $25 \%$ & & \\
\hline RS (\%) & 43.3 & 33.6 & 26.3 & 39.6 & 56.0 \\
\hline RMR (\%) & 46.7 & 43.3 & 40.0 & 46.7 & 76.7 \\
\hline MDR (\%) & 73.2 & 69.6 & 66.1 & 71.4 & 91.1 \\
\hline Total (\%) & 46.0 & 37.1 & 30.2 & 42.6 & 59.8 \\
\hline
\end{tabular}

Note: RS: rifampicin-susceptible

$R M R$ rifampicin-monoresistant

MDR multidrug-resistant

CHE1: catastrophic health expenditure measured by annual out-of-pocket payments exceeding $40 \%$ of the household's annual capacity to pay during

TB treatment

CHE2: catastrophic health expenditure measured by annual out-of-pocket payments exceeding 10\% of the household's annual income during TB treatment 
Table 5 Univariate logistic regression analysis of influencing factors of catastrophic total costs

\begin{tabular}{|c|c|c|c|c|c|}
\hline Variables & $\mathrm{n} / \mathrm{N}$ & $\%$ & OR & $95 \% \mathrm{Cl}$ & $P$-value \\
\hline \multicolumn{6}{|l|}{ Age (years) } \\
\hline$<60$ & $157 / 514$ & 30.5 & 1 & & \\
\hline$\geq 60$ & $92 / 158$ & 58.2 & 3.17 & $2.19,4.58$ & $<0.001$ \\
\hline \multicolumn{6}{|l|}{ Gender } \\
\hline Male & $161 / 432$ & 37.3 & 1 & & \\
\hline Female & $88 / 240$ & 36.7 & 0.97 & $0.70,1.35$ & 0.877 \\
\hline \multicolumn{6}{|l|}{ Education } \\
\hline None & $38 / 70$ & 54.3 & 1 & & \\
\hline Primary or Junior school & $155 / 368$ & 42.1 & 0.61 & $0.37,1.02$ & 0.062 \\
\hline Senior school or above & $56 / 234$ & 23.9 & 0.26 & $0.15,0.46$ & $<0.001$ \\
\hline \multicolumn{6}{|l|}{ Floating population } \\
\hline No & $171 / 437$ & 39.1 & 1 & & \\
\hline Yes & $78 / 235$ & 33.2 & 0.77 & $0.55,1.08$ & 0.129 \\
\hline \multicolumn{6}{|l|}{ Minimum living security household } \\
\hline No & $224 / 640$ & 35 & 1 & & \\
\hline Yes & $25 / 32$ & 78.1 & 6.63 & $2.82,15.58$ & $<0.001$ \\
\hline \multicolumn{6}{|l|}{ Residence } \\
\hline Rural & $123 / 316$ & 38.9 & 1 & & \\
\hline Urban & $126 / 356$ & 35.4 & 0.86 & $0.63,1.18$ & 0.344 \\
\hline \multicolumn{6}{|l|}{ Employment status before illness } \\
\hline Employed & $122 / 422$ & 28.9 & 1 & & \\
\hline Unemployed & $127 / 250$ & 50.8 & 2.54 & $1.83,3.51$ & $<0.001$ \\
\hline \multicolumn{6}{|l|}{ Employment status after illness } \\
\hline Employed & $76 / 286$ & 26.6 & 1 & & \\
\hline Unemployed & $173 / 386$ & 44.8 & 2.24 & $1.61,3.12$ & $<0.001$ \\
\hline \multicolumn{6}{|l|}{ Local basic medical insurance } \\
\hline No & $86 / 212$ & 40.6 & 1 & & \\
\hline Yes & $163 / 460$ & 35.4 & 0.80 & $0.58,1.12$ & 0.201 \\
\hline \multicolumn{6}{|l|}{ Household economic status } \\
\hline Q1 & 19/135 & 14.1 & 1 & & \\
\hline Q2 & $35 / 163$ & 21.5 & 1.67 & $0.90,3.08$ & 0.101 \\
\hline Q3 & $52 / 157$ & 33.1 & 3.02 & $1.68,5.44$ & $<0.001$ \\
\hline Q4 & $49 / 95$ & 51.6 & 6.50 & $3.46,12.21$ & $<0.001$ \\
\hline Q5 & $94 / 122$ & 77.1 & 20.50 & $10.78,38.99$ & $<0.001$ \\
\hline \multicolumn{6}{|l|}{ Patient delay } \\
\hline No & $101 / 312$ & 32.4 & 1 & & \\
\hline Yes & $148 / 360$ & 41.1 & 1.46 & $1.06,2.00$ & 0.020 \\
\hline \multicolumn{6}{|l|}{ Diagnosis delay } \\
\hline No & $213 / 583$ & 36.5 & 1 & & \\
\hline Yes & $36 / 89$ & 40.4 & 1.18 & $0.75,1.86$ & 0.477 \\
\hline \multicolumn{6}{|l|}{ Type of case } \\
\hline New or never treated & $206 / 601$ & 34.3 & 1 & & \\
\hline Relapse or previously treated & $43 / 71$ & 60.6 & 2.94 & $1.78,4.88$ & $<0.001$ \\
\hline
\end{tabular}

Comorbidities 
Table 5 Univariate logistic regression analysis of influencing factors of catastrophic total costs (Continued)

\begin{tabular}{|c|c|c|c|c|c|}
\hline Variables & $\mathrm{n} / \mathrm{N}$ & $\%$ & OR & $95 \% \mathrm{Cl}$ & $P$-value \\
\hline No & $195 / 584$ & 33.4 & 1 & & \\
\hline Yes & $54 / 88$ & 61.4 & 3.17 & $2.00,5.03$ & $<0.001$ \\
\hline \multicolumn{6}{|c|}{ Seeking medical care outside the city } \\
\hline No & $204 / 587$ & 34.8 & 1 & & \\
\hline Yes & $45 / 85$ & 52.9 & 2.11 & $1.34,3.34$ & 0.001 \\
\hline \multicolumn{6}{|c|}{ Hospitalisation } \\
\hline No & $50 / 284$ & 17.6 & 1 & & \\
\hline Yes & 199/388 & 51.3 & 4.93 & $3.42,7.09$ & $<0.001$ \\
\hline \multicolumn{6}{|c|}{ Severe adverse drug reactions } \\
\hline No & $153 / 481$ & 31.8 & 1 & & \\
\hline Yes & $96 / 191$ & 50.3 & 2.17 & $1.54,3.05$ & $<0.001$ \\
\hline \multicolumn{6}{|c|}{ Drug sensitivity test } \\
\hline RS & 197/586 & 33.6 & 1 & & \\
\hline RMR & $13 / 30$ & 43.3 & 1.51 & $0.72,3.17$ & 0.277 \\
\hline MDR & $39 / 56$ & 69.6 & 4.53 & $2.50,8.21$ & $<0.001$ \\
\hline
\end{tabular}

Note: Floating population refers to a group of migrants without Hukou registered in the place where they live or work

Minimum living security household, that is, a household's anuual income per capita is less than US\$ 1429 , must be certified by the local civil affairs department Local basic medical insurance refers to the basic medical insurance registered in Ningbo city

Patient delay is determined as a time interval from the first onset of tuberculosis symptoms to the first visit to any health provider lasts for $>14$ days

Diagnostic delay is defined as a time interval between a patient's first visit to any health provider and the final diagnosis as TB lasts for $>14$ days

Household economic status is ranked based on a household's annual income per capita, with quartile 1 (Q1) being the richest and quartile 5 (Q5) being the poorest

$R S$ rifampicin-susceptible; $R M R$ rifampicin-monoresistant; $M D R$ multidrug-resistant

OR odds ratio; $95 \%$ Cl 95\% confidence intervals

transfers, patients and national TB program are challenged by excessive costs caused by unnecessary hospitalization, out-of-network medical care and inappropriate prescribing patterns. Healthcare facility managers and government administrators should conduct in-depth investigation into the causes of excessive direct medical costs, as well as audit to identify fraud and adverse financial incentives to physicians. Additionally, fee-for-service payment model could have incentivized unnecessary care and overprescribing of certain medications. Value-based payment model with outcomebased contracting with TB treatment services might play a role in reducing overall costs without impacting care quality. Finally, there is reason to believe that the current one-size-fit all subsidy policy may be inadequate for the most financially vulnerable population. Policymakers should consider tailored financial subsidies addition to in-kind support to improve equity of TB programs. On the other hand, means test and asset assessment should be considered in determining program eligibility to improve the efficiency of subsidies programs.

Our study has some limitations. First, the study did not include some patients who did not receive treatment or discontinued treatment prematurely. If this situation is caused by financial barriers, it has the possibility of underestimating the actual level of catastrophic costs.
However, the research still illustrates that patients and their households face huge economic risks. Second, we employed self-reported actual medical costs (cross-referenced by the medical receipts collected by community doctors during each visit) to estimate the cost estimates in this study rather than the method recommended by WHO, which suggests asking these questions while on treatment. We adopted this approach based on the operational reasons since it was logistically challenging to track and survey internal migrant patients on treatment. Our approach may have introduced recall bias in economic outcome measures. However, in the pilot survey, we found that our approach and WHO-recommended method yielded similar cost estimates in a subgroup of study participants. Nevertheless, we acknowledge the recall bias on self-reported direct non-medical expenses, household income and food expenditure due to longer recall time as one of the limitations.

\section{Conclusion}

In Ningbo, households with pulmonary TB patient treated in designated hospitals, especially those with MDR, were facing catastrophic economic risks. There were many independent factors that affected the occurrence of catastrophic costs. Although direct medical costs accounted for most of the catastrophic costs, 
Table 6 Multivariable logistic regression analysis of factors associated with catastrophic total costs

\begin{tabular}{|c|c|c|c|c|c|c|}
\hline \multirow[t]{2}{*}{ Variable } & \multicolumn{3}{|c|}{ Multivariable } & \multicolumn{3}{|c|}{ Multivariable* } \\
\hline & $\mathrm{aOR}$ & $95 \% \mathrm{Cl}$ & $P$-value & $\mathrm{aOR}$ & $95 \% \mathrm{Cl}$ & $P$-value \\
\hline \multicolumn{7}{|l|}{ Age (years) } \\
\hline$<60$ & 1 & & & 1 & & \\
\hline$\geq 60$ & 3.41 & $1.85,6.32$ & $<0.001$ & 4.32 & $2.26,8.27$ & $<0.001$ \\
\hline \multicolumn{7}{|l|}{ Education } \\
\hline None & 1 & & & 1 & & \\
\hline Primary or Junior school & 1.31 & $0.62,2.80$ & 0.480 & 1.31 & $0.62,2.80$ & 0.480 \\
\hline Senior school or above & 1.09 & $0.46,2.62$ & 0.840 & 1.09 & $0.46,2.62$ & 0.840 \\
\hline \multicolumn{7}{|l|}{ Minimum living security household } \\
\hline No & 1 & & & 1 & & \\
\hline Yes & 5.69 & $1.95,16.60$ & 0.001 & 5.69 & $1.95,16.60$ & 0.001 \\
\hline \multicolumn{7}{|l|}{ Employment status before illness } \\
\hline Employed & 1 & & & 1 & & \\
\hline Unemployed & 1.67 & $1.04,2.69$ & 0.036 & 1.67 & $1.04,2.69$ & 0.036 \\
\hline \multicolumn{7}{|l|}{ Employment status after illness } \\
\hline Employed & 1 & & & 1 & & \\
\hline Unemployed & 2.47 & $1.49,4.08$ & $<0.001$ & 2.47 & $1.49,4.08$ & $<0.001$ \\
\hline \multicolumn{7}{|l|}{ Local basic medical insurance } \\
\hline No & 1 & & & 1 & & \\
\hline Yes & 0.52 & $0.32,0.85$ & 0.009 & 0.52 & $0.32,0.85$ & 0.009 \\
\hline \multicolumn{7}{|l|}{ Household economic status } \\
\hline Q1 & 1 & & & 1 & & \\
\hline Q2 & 1.54 & $0.75,3.17$ & 0.242 & 1.54 & $0.75,3.17$ & 0.242 \\
\hline Q3 & 3.00 & $1.48,6.10$ & 0.002 & 3.01 & $1.48,6.10$ & 0.002 \\
\hline Q4 & 7.28 & $3.29,16.10$ & $<0.001$ & 7.26 & $3.28,16.06$ & $<0.001$ \\
\hline Q5 & 15.84 & $7.27,34.55$ & $<0.001$ & 15.91 & $7.28,34.76$ & $<0.001$ \\
\hline \multicolumn{7}{|l|}{ Type of case } \\
\hline New or never treated & 1 & & & 1 & & \\
\hline Relapse or previously treated & 1.35 & $0.70,2.61$ & 0.376 & 1.35 & $0.70,2.61$ & 0.378 \\
\hline \multicolumn{7}{|l|}{ Comorbidities } \\
\hline No & 1 & & & 1 & & \\
\hline Yes & 1.71 & $0.94,3.13$ & 0.080 & 1.71 & $0.94,3.12$ & 0.082 \\
\hline \multicolumn{7}{|c|}{ Seeking medical care outside the city } \\
\hline No & 1 & & & 1 & & \\
\hline Yes & 2.29 & $1.21,4.34$ & 0.011 & 2.29 & $1.21,4.34$ & 0.011 \\
\hline \multicolumn{7}{|l|}{ Hospitalisation } \\
\hline No & 1 & & & 1 & & \\
\hline Yes & 5.42 & $3.35,8.76$ & $<0.001$ & 5.54 & $3.15,9.75$ & $<0.001$ \\
\hline \multicolumn{7}{|l|}{ Severe adverse drug reactions } \\
\hline No & 1 & & & 1 & & \\
\hline Yes & 1.95 & $1.24,3.08$ & 0.004 & 2.05 & $0.90,4.66$ & 0.087 \\
\hline \multicolumn{7}{|l|}{ Drug sensitivity test } \\
\hline RS & 1 & & & 1 & & \\
\hline RMR & 1.15 & $0.45,2.97$ & 0.768 & 1.16 & $0.45,2.99$ & 0.761 \\
\hline
\end{tabular}


Table 6 Multivariable logistic regression analysis of factors associated with catastrophic total costs (Continued)

\begin{tabular}{|c|c|c|c|c|c|c|}
\hline \multirow[t]{2}{*}{ Variable } & \multicolumn{3}{|c|}{ Multivariable } & \multicolumn{3}{|c|}{ Multivariable* } \\
\hline & $\mathrm{aOR}$ & $95 \% \mathrm{Cl}$ & $P$-value & $\mathrm{aOR}$ & $95 \% \mathrm{Cl}$ & $P$-value \\
\hline MDR & 2.78 & $1.28,6.02$ & 0.010 & 2.77 & $1.27,6.01$ & 0.010 \\
\hline
\end{tabular}

Note: Minimum living security household is defined as a household's anuual income per capita lower than US\$1429. The designation of minimum living security household status needs to be certified by the local civil affairs bureau

Local basic medical insurance refers to the basic medical insurance registered in Ningbo city

Household economic status is ranked based on a household's annual income per capita, with quartile 1 (Q1) being the richest and quartile 5 (Q5) being the poorest

Comorbidities refers to the simultaneous occurrence of malignancy, diabetes mellitus, renal disease, etc.

$R S$ rifampicin-susceptible; $R M R$ rifampicin-monoresistant; $M D R$ multidrug-resistant

aOR Adjusted odds ratio; 95\% Cl 95\% confidence intervals

*The multivariable logistic model accounted the interaction between hospitalization and severe adverse drug reactions

protecting patients from financial disasters requires not only expanding universal health coverage, but also more personalized economic protection measures such as cash transfers to compensate low-income households for direct non-medical expenses and income loss. In addition, attention should be paid to payment model reforms aimed at reducing unnecessary hospitalization.

\section{Abbreviations}

TB: Tuberculosis; CHE: Catastrophic Health Expenditure; WHO: World Health Organization; NTCP: National Tuberculosis Control Program; OOP: Out-ofpocket; RS: Rifampicin-Susceptible; RMR: Rifampicin-Monoresistant; MDR: Multidrug-Resistant; LASSO: Least Absolute Shrinkage and Selection Operator

\section{Acknowledgements}

We sincerely thank all the participants in this project. We appreciate Dr. Jifang Zhou's assistance on manuscript writing.

\section{Authors' contributions}

TY participated in the design of the study, performed the analysis and interpretation of the data, drafted and revised the manuscript. TC participated in the analysis and interpretation of the data. YC, QC and DB participated in the collection of data, revised the manuscript. All authors read and approved the final version of the manuscript.

\section{Funding}

This research was supported by Zhejiang Medical Research Project (2018KY733). The funder had no role in study design, data collection, analysis, interpretation of data and writing the manuscript.

\section{Availability of data and materials}

Please contact the corresponding author for data requests.

\section{Ethics approval and consent to participate}

The study was approved by the Research Ethics Committee of Ningbo Municipal Center for Disease Control and Prevention, China. All the participants read and signed the informed consent forms.

\section{Consent for publication}

Not applicable.

\section{Competing interests}

The authors declare that they have no competing interests.

\section{Author details}

'Institute of Tuberculosis Prevention and Control, Ningbo Municipal Center for Disease Control and Prevention, Ningbo, Zhejiang, China. ${ }^{2}$ Division of Medical Insurance, Ningbo Huamei Hospital, University of Chinese Academy of Sciences, Ningbo, China. ${ }^{3}$ Institute of Infectious Disease Control, Haishu District Center for Disease Control and Prevention, Ningbo, Zhejiang, China.
Received: 27 January 2020 Accepted: 17 June 2020

Published online: 26 June 2020

\section{References}

1. Global tuberculosis report 2018 [https:/apps.who.int/iris/bitstream/handle/1 0665/274453/9789241565646-eng.pdf?ua =1\&ua=1].

2. Shete PB, Reid M, Goosby E. Message to world leaders: we cannot end tuberculosis without addressing the social and economic burden of the disease. Lancet Glob Health. 2018;6(12):e1272-3.

3. Uplekar M, Weil D, Lonnroth K, Jaramillo E, Lienhardt C, Dias HM, Falzon D, Floyd K, Gargioni G, Getahun H, et al. WHO's new end TB strategy. Lancet. 2015;385(9979):1799-801.

4. Wang L, Liu J, Chin DP. Progress in tuberculosis control and the evolving public-health system in China. Lancet. 2007;369(9562):691-6.

5. Liu Q, Smith H, Wang Y, Tang S, Wang Q, Garner P. Tuberculosis patient expenditure on drugs and tests in subsidised, public services in China: a descriptive study. Tropical Med Int Health. 2010;15(1):26-32.

6. Long Q, Smith H, Zhang T, Tang S, Garner P. Patient medical costs for tuberculosis treatment and impact on adherence in China: a systematic review. BMC Public Health. 2011;11:393.

7. Liu X, Thomson R, Gong Y, Zhao F, Squire SB, Tolhurst R, Zhao X, Yan F, Tang S. How affordable are tuberculosis diagnosis and treatment in rural China? An analysis from community and tuberculosis patient perspectives, Tropical Med Int Health. 2007;12(12):1464-71.

8. Duan W, Zhang W, Wu C, Wang Q, Yu Y, Lin H, Liu Y, Hu D. Extent and determinants of catastrophic health expenditure for tuberculosis care in Chongqing municipality, China: a cross-sectional study. BMJ Open. 2019;9(4): e026638.

9. Zhou C, Long Q, Chen J, Xiang L, Li Q, Tang S, Huang F, Sun Q, Lucas H. Factors that determine catastrophic expenditure for tuberculosis care: a patient survey in China. Infect Dis Poverty. 2016;5:6.

10. Xiang L, Pan Y, Hou S, Zhang H, Sato KD, Li Q, Wang J, Tang S. The impact of the new cooperative medical scheme on financial burden of tuberculosis patients: evidence from six counties in China. Infect Dis Poverty. 2016;5:8.

11. Tuberculosis patient cost surveys: a handbook [https://www.who.int/tb/ publications/patient cost surveys/en/].

12. Xu K, Evans DB, Kawabata K, Zeramdini R, Klavus J, Murray CJ. Household catastrophic health expenditure: a multicountry analysis. Lancet. 2003: 362(9378):111-7.

13. Ukwaja KN, Alobu I, Abimbola S, Hopewell PC. Household catastrophic payments for tuberculosis care in Nigeria: incidence, determinants, and policy implications for universal health coverage. Infect Dis Poverty. 2013; 2(1):21.

14. Wingfield T, Tovar MA, Huff D, Boccia D, Montoya R, Ramos E, Lewis JJ, Gilman RH, Evans CA. The economic effects of supporting tuberculosisaffected households in Peru. Eur Respir J. 2016;48(5):1396-410.

15. Pedrazzoli D, Siroka A, Boccia D, Bonsu F, Nartey K, Houben R, Borghi J. How affordable is TB care? Findings from a nationwide TB patient cost survey in Ghana. Tropical Med Int Health. 2018;23(8):870-8.

16. Nhung NV, Hoa NB, Anh NT, Anh LTN, Siroka A, Lonnroth K, Garcia Baena I. Measuring catastrophic costs due to tuberculosis in Viet Nam. Int J Tuberc Lung Dis. 2018;22(9):983-90.

17. Fuady A, Houweling TAJ, Mansyur M, Richardus JH. Catastrophic total costs in tuberculosis-affected households and their determinants since 
Indonesia's implementation of universal health coverage. Infect Dis Poverty. 2018;7(1):3.

18. Wingfield T, Boccia D, Tovar M, Gavino A, Zevallos K, Montoya R, Lonnroth $K$, Evans CA. Defining catastrophic costs and comparing their importance for adverse tuberculosis outcome with multi-drug resistance: a prospective cohort study, Peru. PLoS Med. 2014;11(7):e1001675.

19. Muniyandi M, Thomas BE, Karikalan N, Kannan T, Rajendran K, Saravanan B, Vohra V, Okorosobo T, Lonnroth K, Tripathy SP. Association of Tuberculosis with Household Catastrophic Expenditure in South India. JAMA Netw Open. 2020;3(2):e1920973.

20. Jiang WX, Long Q, Lucas H, Dong D, Chen JY, Xiang L, Li Q, Huang F, Wang $\mathrm{H}$, Elbers $\mathrm{C}$, et al. Impact of an innovative financing and payment model on tuberculosis patients' financial burden: is tuberculosis care more affordable for the poor? Infect Dis Poverty. 2019;8(1):21.

21. Boccia D, Pedrazzoli $D$, Wingfield T, Jaramillo E, Lonnroth $K$, Lewis J, Hargreaves J, Evans CA. Towards cash transfer interventions for tuberculosis prevention, care and control: key operational challenges and research priorities. BMC Infect Dis. 2016;16:307.

22. Hu H, Chen J, Sato KD, Zhou Y, Jiang H, Wu P, Wang H. Factors that associated with TB patient admission rate and TB inpatient service cost: a cross-sectional study in China. Infect Dis Poverty. 2016;5:4.

23. Jia X, Chen J, Zhang S, Dai B, Long Q, Tang S. Implementing a "free" tuberculosis (TB) care policy under the integrated model in Jiangsu, China: practices and costs in the real world. Infect Dis Pov. 2016;5:1.

24. Qiu S, Pan H, Zhang S, Peng X, Zheng X, Xu G, Wang M, Wang J, Lu H. Is tuberculosis treatment really free in China? A study comparing two areas with different management models. PLoS One. 2015;10(5):e0126770.

25. Wei X, Zou G, Yin J, Walley J, Zhang X, Li R, Sun Q. Effective reimbursement rates of the rural health insurance among uncomplicated tuberculosis patients in China. Tropical Med Int Health. 2015;20(3):304-11.

26. Hu D, Liu X, Chen J, Wang Y, Wang T, Zeng W, Smith H, Garner P. Direct observation and adherence to tuberculosis treatment in Chongqing, China: a descriptive study. Health Policy Plan. 2008;23(1):43-55.

27. Xu B, Dong HJ, Zhao Q, Bogg L. DOTS in China - removing barriers or moving barriers? Health Policy Plan. 2006;21(5):365-72.

28. Zhan S, Wang L, Yin A, Blas E. Revenue-driven in TB control--three cases in China. Int J Health Plann Manag. 2004;19(Suppl 1):S63-78.

\section{Publisher's Note}

Springer Nature remains neutral with regard to jurisdictional claims in published maps and institutional affiliations.

Ready to submit your research? Choose BMC and benefit from:

- fast, convenient online submission

- thorough peer review by experienced researchers in your field

- rapid publication on acceptance

- support for research data, including large and complex data types

- gold Open Access which fosters wider collaboration and increased citations

- maximum visibility for your research: over $100 \mathrm{M}$ website views per year

At BMC, research is always in progress.

Learn more biomedcentral.com/submissions 